\title{
An Investigation into the Factors Influencing Drug Release from Hydrophilic Matrix Tablets Based on Novel Carbomer Polymers
}

Jelena Parojčić, Zorica Đurić, Milica Jovanović \& Svetlana Ibrić

To cite this article: Jelena Parojčić, Zorica Đurić, Milica Jovanović \& Svetlana Ibrić (2004) An Investigation into the Factors Influencing Drug Release from Hydrophilic Matrix Tablets Based on Novel Carbomer Polymers, Drug Delivery, 11:1, 59-65, DOI: 10.1080/10717540490265379

To link to this article: https://doi.org/10.1080/10717540490265379

Published online: 19 Oct 2008.

Submit your article to this journal $\sqsubset$

Џ Article views: 305

Citing articles: 22 View citing articles $\square$ 


\title{
An Investigation into the Factors Influencing Drug Release from Hydrophilic Matrix Tablets Based on Novel Carbomer Polymers
}

\author{
Jelena Parojčić, Zorica Đurić, Milica Jovanović, and Svetlana Ibrić \\ Institute of Pharmaceutical Technology, Faculty of Pharmacy, Belgrade, Yugoslavia
}

\begin{abstract}
Drug release from hydrophilic matrix tablets can be strongly influenced by the proportion of matrix forming polymer and the dimensions and geometry of the tablets. A complete two-factor, three-level factorial design, followed by multiple regression analysis and response surface methodology, was applied to investigate the influence of polymer level and tablet size on drug release kinetics from hydrophilic matrix tablets prepared with Carbopol 971P and Carbopol 71G. Tablet diameter, radius-to-height ratio, tablet surface area, and surface-area-to-volume ratio were evaluated as independent variables in terms of their applicability to characterize tablet size and geometry. The results indicate that it may be possible to control the rate of drug release by modifying the proportion of carbomer in tablets and tablet dimensions. The practical benefit of these simulations is to optimize the geometry and dimensions of a controlled release device and reduce the number of experiments involved in the development of new controlled release dosage forms.
\end{abstract}

Keywords Carbomer, Dissolution, Drug Delivery, Hydrophilic Matrix Tablet, Paracetamol, Sustained Release

Hydrophilic matrix tablets are among the most widely used controlled release dosage forms for oral delivery due to their low cost and ease of fabrication. Drug release from hydrophilic matrix tablets upon contact with dissolution media or physiological fluids involves hydratation of tablet surface and formation of the gel layer that swells imbibing additional amount of water. The dissolved drug diffuses through the gel layer and hydratation and swelling progress into the tablet core. It could be expected that these processes are dependent on the type and proportion of the polymer used as a controlled release agent, as well as the tablet geometry and dimensions. The subject of tablet size and shape should be considered both from the physiological and the manufacturers point of view.

Received 26 January 2003; accepted 19 May 2003.

Address correspondence to Jelena Parojčić, Institute of Pharmaceutical Technology, Faculty of Pharmacy, Vojvode Stepe 450, 11221 Belgrade, Yugoslavia. E-mail: sptasha@ptt.yu
Channer and Virjee (1986) investigated the influence of tablet size and shape on transit time through the esophagus and found that large oblong tablets had significantly lower transit time compared with large plain tablets, whereas the transit time of small and medium tablets was lower than that for large tablets irrespective of their shape. Also, the residence time of dosage form in stomach could depend on the size of the dosage form (Wilson 1998). From the formulators point of view, tablet size and shape should be evaluated regarding required dose of the active substance, content of excipients, mechanical characteristics of the tablets, accessibility of tableting tools of different sizes and shapes, and above all the targeted drug release profile.

Several researchers (Siepmann et al. 1998, 2000; Siepmann, Streubel, and Peppas 2002; Reynolds, Mitchell, and Balwinski 2002; Karasulu, Ertan, and Kose 2000; Kim 1995) investigated the effect of matrix geometry on drug release from sustained release dosage forms. They have mostly focused on application and evaluation of hydroxypropylmethylcellulose (HPMC) as a controlled release agent. The selection of the parameter that appropriately characterizes the tablet geometry is questionable. Tablet size is usually expressed by the tablet radius or tablet weight, but it appears that tablet height as well as the tablet surface area and volume should be evaluated. Siepmann et al. (2000) examined the effect of the aspect ratio (radius/height) and the size of cylindrical matrices on drug release and reported that release from small tablets is faster than from large cylindrical tablets due to the higher relative surface area (absolute surface area/absolute volume). Reynolds et al. (2002) studied the effect of relative surface area of the tablets on drug release pattern and concluded that this parameter is valuable when comparing relative drug release from tablets of varying shapes.

Considering the apparent differences in the behavior of hydrophilic matrices prepared with HPMC and carbomer polymers upon contact with dissolution medium, i.e., the extensive swelling of carbomer matrices, the effect of polymer concentration, and matrix geometry for tablets based on carbomer resins should be evaluated. Some authors evaluated the effect 
of polymer level on drug release, from carbomer matrix tablets, without considering the effect of tablet size (Khan and Zhu 1999; Huang and Schwartz 1995; Fereira et al. 1995; Perez-Marcos et al. 1995).

Carbomers, synthetic high molecular weight acrylic acid polymers cross-linked with polyalkenyl polyether and commercially available under the brand name of Carbopol ${ }^{\circledR}$, are not new in sustained release technology but are still interesting. Most of the work done on the evaluation of carbomers used the Carbopol 934 and Carbopol 974P polymers. Novel, toxicologically preferred Carbopol 971P and technologically improved Carbopol $71 \mathrm{G}$ polymers were used in this study.

The nonsalicylate analgesic and antipyretic drug, paracetamol, which is voluminous and poorly compressible, was used as a model substance. Paracetamol is generally classified as a drug with high solubility that shows high permeability throughout the intestinal tract, meeting the criteria as a class I drug according to the Biopharmaceutics Classification System (Amidon and Lobenberg 2000). The absorption of class I drugs should depend on their in vivo dissolution, controlled by the kinetics of drug release from the dosage form.

To statistically evaluate the effect of polymer content and tablet size, factorially designed experiments were conducted, followed by multiple regression analysis and response surface methodology (Box, Hunter, and Hunter 1978; Montgomery 1997).

\section{MATERIALS AND METHODS}

The following materials were obtained from commercial suppliers and used as received: paracetamol (Merck, Germany), Carbopol 971P, Carbopol 71G (BFGoodrich, USA), lactose monohydrate (Fluka Chemie, Switzerland), polivinylpirolidone (Kollidon 30, BASF, Germany), microcrystalline cellulose (Emcocell XLM 90, Penwest, USA), collodial silicium dioxide (Aerosil 200, Degussa, Germany), and sodium stearyl fumarate (Pruv, Penvest, USA).

\section{Experimental Design}

Two separate experimental runs including 9 samples were performed according to the $3^{2}$ experimental design. Additionally, the central experiment was done in triplicate to determine validity of the obtained mathematical models. The independent variables were proportion of Carbopol 971P or Carbopol $71 \mathrm{G}\left(\mathrm{X}_{1}\right)$ and tablet size $\left(\mathrm{X}_{2}\right)$. The proportion of polymer was $7.5,15.0$, or $22.5 \%$. Tablet size was expressed as tablet radius, radius-to-height ratio (aspect ratio), tablet surface area, and surface-area-to-volume ratio (designated as relative surface area). The selected response variable ( $\mathrm{Y}$ ) was percent of drug released after $5 \mathrm{hr}$ of investigation $\left(\mathrm{D}_{5 \mathrm{~h}}\right)$. The experimental matrix, real and coded values of investigated factors, and response variable are presented in Tables 1 and 2 for samples prepared with Carbopol 971P and Carbopol 71G, respectively.

After performing the experiments, multiple linear regression was applied to evaluate the regression coefficients of the mathematical model that included the linear and quadratic terms of both factors investigated, as well as the interaction factor:

$$
\mathrm{Y}=\mathrm{b}_{0}+\mathrm{b}_{1} \mathrm{X}_{1}+\mathrm{b}_{2} \mathrm{X}_{2}+\mathrm{b}_{3} \mathrm{X}_{1}^{2}+\mathrm{b}_{4} \mathrm{X}_{2}^{2}+\mathrm{b}_{5} \mathrm{X}_{1} \mathrm{X}_{2}
$$

Student's $t$-test was applied to each term of the quadratic model to evaluate their significance. Only terms that were significant were included in the final model. An experimental matrix was, after the estimation of regression coefficients, used to characterize the results by response surface methodology. Response surface and contour plots were drawn to visualize the effect of investigated factors.

\section{Preparation of Tablets}

Tablet samples were prepared using conventional wet granulation method. A paracetamol-lactose mixture (80:20) was granulated using $5 \%$ polyvinylpyrrolidone aqueous dispersion. The sustained release agent was added extragranularly, as well as

TABLE 1

Experimental matrix with the real and coded values of investigated factors and response variable for samples prepared with Carbopol 971P

Factor levels

\begin{tabular}{|c|c|c|c|c|c|c|c|c|c|c|c|}
\hline \multirow{3}{*}{ Sample } & \multicolumn{2}{|c|}{$\mathrm{X}_{1}$} & \multirow{3}{*}{ Coded } & \multicolumn{7}{|c|}{$\mathrm{X}_{2}$} & \multirow{3}{*}{$\begin{array}{c}\mathrm{Y} \\
\mathrm{D}_{5 \mathrm{~h}}(\%)\end{array}$} \\
\hline & \multirow[t]{2}{*}{ Coded } & \multirow[t]{2}{*}{ Real } & & \multicolumn{7}{|c|}{ Real } & \\
\hline & & & & $\mathrm{m}(\mathrm{mg})$ & $\mathrm{d}(\mathrm{mm})$ & $\mathrm{h}(\mathrm{mm})$ & $\mathrm{r} / \mathrm{h}$ & $\mathrm{P}\left(\mathrm{mm}^{2}\right)$ & $\mathrm{V}\left(\mathrm{mm}^{3}\right)$ & $\mathrm{P} / \mathrm{V}$ & \\
\hline P1 & -1 & 7.5 & -1 & 275 & 9 & 4.24 & 1.0613 & 246.99 & 269.60 & 0.9161 & 96.08 \\
\hline P2 & 0 & 15 & -1 & 275 & 9 & 4.26 & 1.0563 & 247.56 & 270.87 & 0.9139 & 66.24 \\
\hline P3 & 1 & 22.5 & -1 & 275 & 9 & 4.66 & 0.9657 & 259.00 & 296.62 & 0.8736 & 50.51 \\
\hline P4 & -1 & 7.5 & 0 & 550 & 12 & 4.39 & 1.3667 & 391.50 & 496.24 & 0.7889 & 44.73 \\
\hline P5 & 0 & 15 & 0 & 550 & 12 & 4.46 & 1.3453 & 394.13 & 504.16 & 0.7817 & 37.78 \\
\hline P6 & 1 & 22.5 & 0 & 550 & 12 & 4.96 & 1.2097 & 412.97 & 560.68 & 0.7365 & 28.79 \\
\hline $\mathrm{P} 7$ & -1 & 7.5 & 1 & 825 & 15 & 4.44 & 1.6892 & 562.37 & 784.22 & 0.7171 & 43.12 \\
\hline P8 & 0 & 15 & 1 & 825 & 15 & 4.60 & 1.6304 & 569.91 & 812.48 & 0.7014 & 34.75 \\
\hline P9 & 1 & 22.5 & 1 & 825 & 15 & 4.88 & 1.5369 & 583.10 & 861.93 & 0.6765 & 26.28 \\
\hline
\end{tabular}


TABLE 2

Experimental matrix with the real and coded values of investigated factors and response variable for samples prepared with Carbopol $71 \mathrm{G}$

\begin{tabular}{|c|c|c|c|c|c|c|c|c|c|c|c|}
\hline \multicolumn{12}{|c|}{ Factor levels } \\
\hline \multirow{3}{*}{ Sample } & \multirow{3}{*}{$\begin{array}{c}\mathrm{X} \\
\text { Coded }\end{array}$} & \multirow{3}{*}{ Real } & \multirow{3}{*}{ Coded } & \multirow{2}{*}{\multicolumn{7}{|c|}{$\mathrm{X}_{2}$}} & \multirow{3}{*}{$\begin{array}{c}\mathrm{Y} \\
\mathrm{D}_{5 \mathrm{~h}}(\%)\end{array}$} \\
\hline & & & & & & & & & & & \\
\hline & & & & $\mathrm{m}(\mathrm{mg})$ & $\mathrm{d}(\mathrm{mm})$ & $\mathrm{h}(\mathrm{mm})$ & $\mathrm{r} / \mathrm{h}$ & $\mathrm{P}\left(\mathrm{mm}^{2}\right)$ & $\mathrm{V}\left(\mathrm{mm}^{3}\right)$ & $\mathrm{P} / \mathrm{V}$ & \\
\hline G1 & -1 & 7.5 & -1 & 275 & 9 & 3.85 & 1.1688 & 235.97 & 244.80 & 0.9639 & 97.52 \\
\hline G2 & 0 & 15 & -1 & 275 & 9 & 3.85 & 1.1688 & 235.97 & 244.80 & 0.9639 & 74.30 \\
\hline G3 & 1 & 22.5 & -1 & 275 & 9 & 3.9 & 1.1538 & 237.38 & 247.98 & 0.9573 & 47.68 \\
\hline G4 & -1 & 7.5 & 0 & 550 & 12 & 4.45 & 1.3483 & 393.76 & 503.03 & 0.7828 & 47.79 \\
\hline G5 & 0 & 15 & 0 & 550 & 12 & 4.5 & 1.3333 & 395.64 & 508.68 & 0.7778 & 37.15 \\
\hline G6 & 1 & 22.5 & 0 & 550 & 12 & 4.55 & 1.3187 & 397.52 & 514.33 & 0.7729 & 27.90 \\
\hline G7 & -1 & 7.5 & 1 & 825 & 15 & 4.49 & 1.6704 & 564.73 & 793.05 & 0.7121 & 45.69 \\
\hline G8 & 0 & 15 & 1 & 825 & 15 & 4.44 & 1.6892 & 562.37 & 784.22 & 0.7171 & 31.97 \\
\hline G9 & 1 & 22.5 & 1 & 825 & 15 & 4.4 & 1.7045 & 560.49 & 777.15 & 0.7212 & 27.30 \\
\hline
\end{tabular}

other excipients, mixed and compressed in an excenter tablet machine (Erweka Korsch EK0, Germany) using flat round punches of various diameters $(9,12$, and $15 \mathrm{~mm})$. The compression pressure was adjusted to provide tablets with hardness between 50 and $60 \mathrm{kN}$. The set of tablet samples prepared with Carbopol 971P was designated as samples P1-P9, while tablet formulations containing Carbopol $71 \mathrm{G}$ were designated as samples G1G9. To evaluate the effect of tablet height, additional samples of tablets containing $22.5 \%$ of Carbopol $71 \mathrm{G}$, with diameters of $12 \mathrm{~mm}$ and $3 \mathrm{~mm}$ (sample G63), $5 \mathrm{~mm}$ (sample G65), and $6 \mathrm{~mm}$ (sample G66) tablet height were prepared.

\section{Drug Release Study}

The release experiments were conducted in the rotating paddle apparatus (Erweka DT 70, Germany), in $1000 \mathrm{ml}$ of distilled water $37^{\circ} \mathrm{C}$, at $50 \mathrm{rpm}$. Then 3 -ml samples were withdrawn at $1 \mathrm{hr}$ intervals, filtered, properly diluted, and assayed UVspectrophotometrically (spectrophotometer Cary 50, Varian, Australia) at $243 \mathrm{~nm}$. Six tablets were studied for each formulation. Upon formation of the gel layer at the tablet surface, tablets stuck to the vessel of the dissolution apparatus, which significantly decreased the drug release rate as the surface area of the matrix exposed to the medium was reduced. To prevent such sticking, wire helix was used that did not restrict the contact of the tablet with the dissolution medium.

Drug release kinetics were evaluated using the following exponential equation proposed by Ritger and Peppas (1987):

$$
\mathrm{M}_{\mathrm{t}} / \mathrm{M}_{\infty}=\mathrm{kt}^{\mathrm{n}}
$$

where $M_{t} / M_{\infty}$ is the fraction of drug released at time $t, k$ is the kinetic constant (drug release rate constant), which incorporates structural and geometrical characteristics of the controlled release system, and $\mathrm{n}$ is the diffusional release exponent indicative of the release mechanism. The experimental data were fitted to the proposed equation using nonlinear regression (LevenbergMarquardt algorithm, Microcal Origin 5.0).

\section{RESULTS AND DISCUSSION}

Drug release profiles obtained for tablet samples prepared with Carbopol 971P (samples P1-P9) and Carbopol 71G (samples G1-G9) are presented in Figures 1 and 2, respectively. More homogenous and uniform matrix swelling was observed in the tablets prepared with Carbopol 971P, whereas with Carbopol $71 \mathrm{G}$ matrices, the disentanglement of polymer chains was much more pronounced as well as more intensive swelling in axial direction. An overall trend of more sustained drug release could be observed in Carbopol 971P tablets during the first hours of investigation, while Carbopol $71 \mathrm{G}$, used as a controlled release agent, tended to maintain the integrity of the matrix and thus prolong drug release. Complete drug release (i.e., $>80 \%$ ) during $8 \mathrm{hr}$ of investigation was observed in "small" tablets and medium and large tablets containing $7.5 \%$ of Carbopol $71 \mathrm{G}$. The drug release was prolonged over $10 \mathrm{hr}$ in medium and large tablets containing $22.5 \%$ of Carbopol $71 \mathrm{G}$ and medium and large tablets containing both $15 \%$ or $22.5 \%$ of Carbopol 971P.

The obtained values of diffusional exponent (n) varied from 1.1818 to 2.2613 for tablets prepared with Carbopol 971P and from 1.5412 to 2.6443 for Carbopol $71 \mathrm{G}$ tablets. Considering the cylindrical shape of the tablets, the $n$ value of 0.89 indicates the zero-order drug release kinetics, while $n>0.89$ denotes super case II transport (Ritger and Peppas 1987). According to the obtained $n$ values, the drug release kinetics could be described as swelling and relaxation of the polymer controlled following the super case II transport.

Multiple regression analysis and analysis of variance revealed that within the investigated experimental field, the effect of both polymer content and tablet size significantly influenced the 


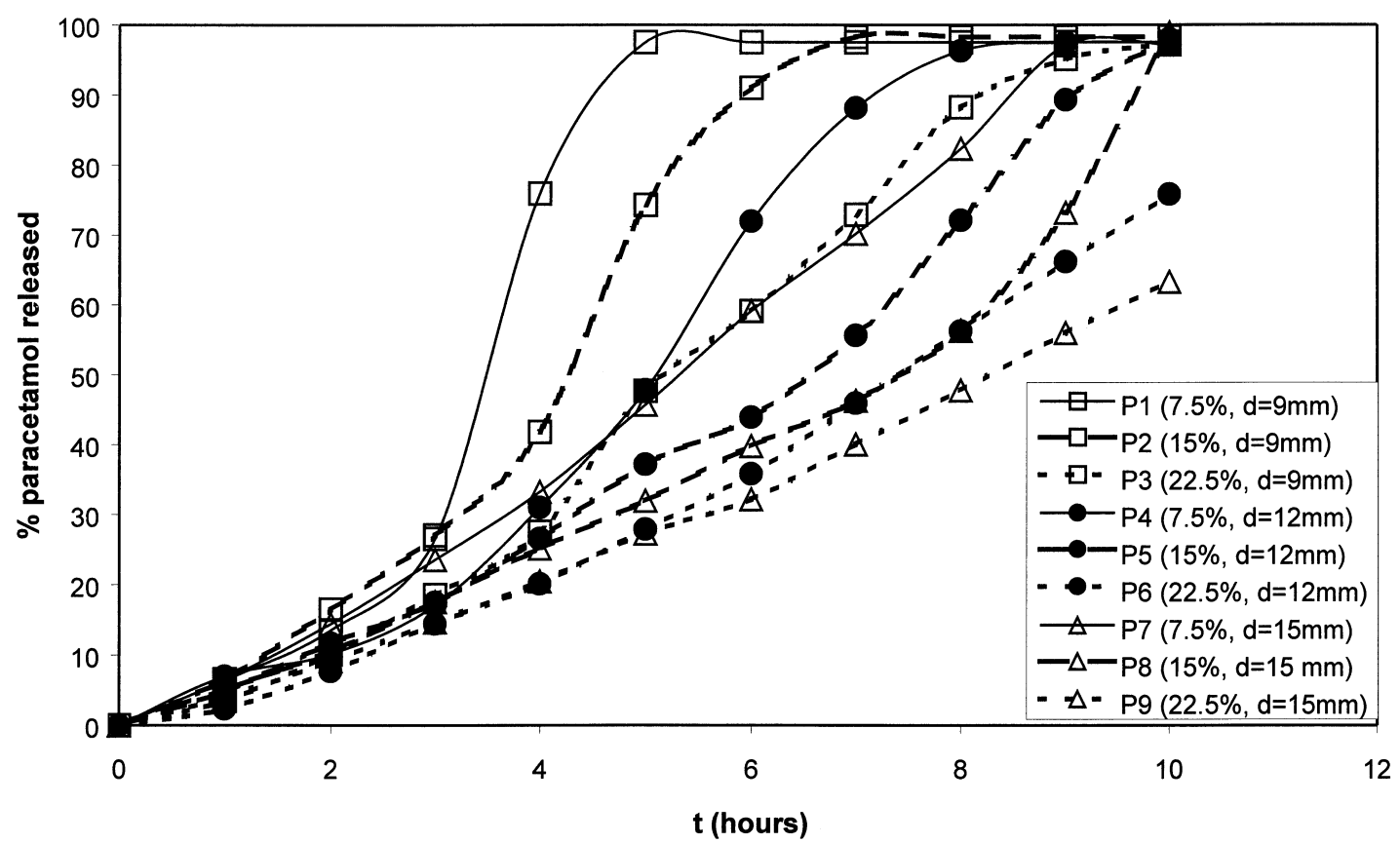

FIG. 1. Drug release profiles obtained for tablets with varying polymer content and tablet size prepared with Carbopol 971P (samples P1-P9).

percent of drug released after $5 \mathrm{hr}$ of investigation. The effect of concentration was linear, while the tablet size exhibited both the linear and quadratic effect. The effect of interaction factor also was significant in the majority of studied cases. The regression coefficients of quadratic models calculated by multiple regression were found to be significant $(p<.05)$ and coefficients of determination for each model are reported in Tables 3 and 4 for series of tablets prepared with Carbopol 971P and Carbopol $71 \mathrm{G}$, respectively. The highest coefficient of determination for the samples prepared with Carbopol 971P was obtained when aspect ratio was selected as the parameter of tablet size, while for the samples prepared with Carbopol $71 \mathrm{G}$, the best fit was obtained for model in which relative surface area was used to characterize the tablet size.

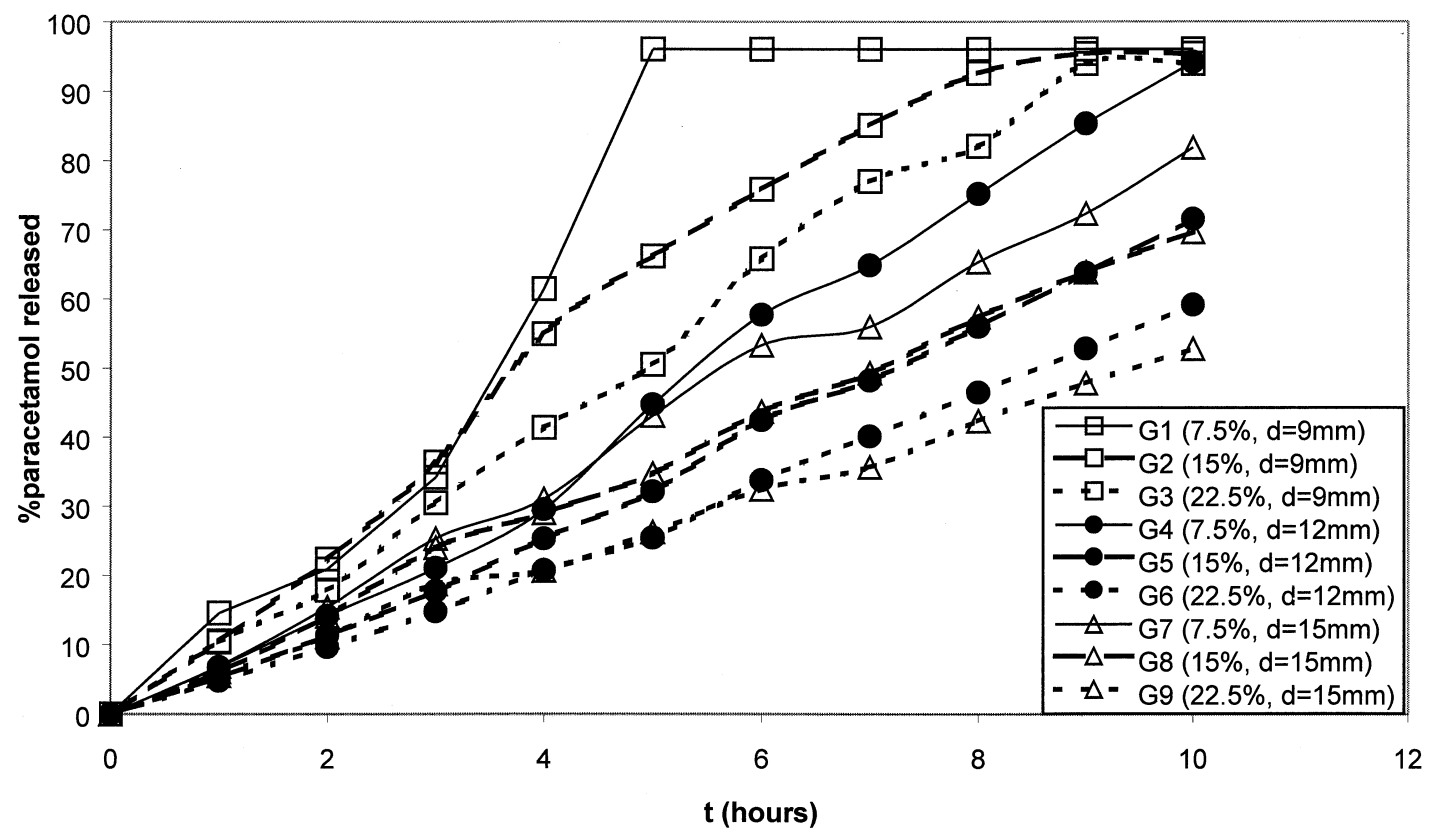

FIG. 2. Drug release profiles obtained for tablets with varying polymer content and tablet size prepared with Carbopol 71G (samples G1-G9). 
TABLE 3

Summary of the multiple regression analysis for tablet samples prepared with Carbopol 971P (samples P1-P9)

\begin{tabular}{lcccc}
\hline \multirow{2}{*}{$\begin{array}{l}\text { Regression } \\
\text { coefficient }\end{array}$} & \multicolumn{4}{c}{ Tablet dimensions evaluated as $\mathrm{X}_{2}$ factor } \\
\cline { 2 - 5 } & Diameter & Radius/height & Surface area & Surface area/volume \\
\hline b0 & 491.187 & 670.294 & 242.147 & 845.330 \\
b1 & -5.647 & -10.590 & -1.727 & -1.43 \\
b2 & -60.817 & -755.649 & -0.783 & -2140.310 \\
b3 & - & - & - & - \\
b4 & 2.083 & 226.373 & 0.0008 & 1446.60 \\
b5 & 0.319 & 6.373 & - & - \\
$\mathrm{R}^{2}$ & 0.9789 & 0.9938 & 0.9402 & 0.9507 \\
\hline
\end{tabular}

The mathematical models obtained for these two sets of tablets, based on tablet diameter and tablet surface area, were almost identical in regard to the calculated set of regression coefficients. Although high coefficients of determination were obtained for model based on the tablet diameter as parameter describing the tablet size, it would not allow the accurate prediction of drug release from matrices of different height. Thus, aspect ratio and relative surface area as parameters encountering both tablet diameter and height should be further evaluated.

The response surface and contour plots of drug released after $5 \mathrm{hr}$ versus polymer content versus tablets aspect ratio for set of tablets prepared with Carbopol 971P and Carbopol 71G are given in Figures 3a and 4a, respectively. Accordingly, the relationship between percent of drug released after $5 \mathrm{hr}$ versus polymer content versus relative surface area of the tablets is presented through the response surface and contour plots presented in Figures $3 b$ and $4 b$, for the two set of tablets.

The drug release profiles observed when studying the effect of tablet height are presented in Figure 5. Although the percent of drug released from samples with various tablet heights (and weights) were different as illustrated in Figure 5b, the actual amounts of drug released were almost identical during the first $5 \mathrm{hr}$ of investigation (Figure 5a). These results were inconsistent with those reported by Siepmann et al. (2000) for hydroxypropylmethylcellulose matrix tablets, in which, with respect to variable tablet height, similar results were obtained for percent of drug released, while the amounts of drug released were markedly different.

In the case of "thin" $(\mathrm{h}=3 \mathrm{~mm})$ tablets, complete drug release and disintegration of matrix occurred after $6 \mathrm{hr}$, while in the case of "thicker" tablets, with $5 \mathrm{~mm}$ and $6 \mathrm{~mm}$ tablet height, drug release proceeded until $12 \mathrm{hr}$ or $14 \mathrm{hr}$ of investigation, respectively.

These results indicate that in the case of Carbopol $71 \mathrm{G}$ matrix tablets, within the usual range of radius/height ratio, the effect of more intensive axial than radial swelling resulted in superimposable drug release profiles during first hours of investigation, regardless of tablet height. However, tablet height was the factor determining the time interval in which the integrity of the matrix remained intact, thus influencing the overall sustained release period.

To evaluate the predictability of the obtained mathematical models, the internal and external predictability values were assessed according to the percent prediction error:

$$
\% \mathrm{PE}=\left[\left|\mathrm{Y}_{\mathrm{obs}}-\mathrm{Y}_{\text {pred }}\right| / \mathrm{Y}_{\mathrm{obs}}\right] \times 100
$$

TABLE 4

Summary of the multiple regression analysis for tablet samples prepared with Carbopol 71G (samples G1-G9)

\begin{tabular}{lcccc}
\hline \multirow{2}{*}{$\begin{array}{l}\text { Regression } \\
\text { coefficient }\end{array}$} & \multicolumn{4}{c}{ Tablet dimensions evaluated as $\mathrm{X}_{2}$ factor } \\
\cline { 2 - 5 } & Diameter & Radius/height & Surface area & Surface area/volume \\
\hline b0 & 469.660 & 956.440 & 253.212 & 314.849 \\
b1 & -6.152 & -2.250 & -4.463 & 5.121 \\
b2 & -55.507 & -1183.350 & -0.726 & -853.553 \\
b3 & - & - & - & - \\
b4 & 1.829 & 389.51 & 0.0006 & 676.723 \\
b5 & 0.349 & - & 0.0064 & -8.571 \\
$\mathrm{R}^{2}$ & 0.9808 & 0.8769 & 0.9599 & 0.9866 \\
\hline
\end{tabular}


a

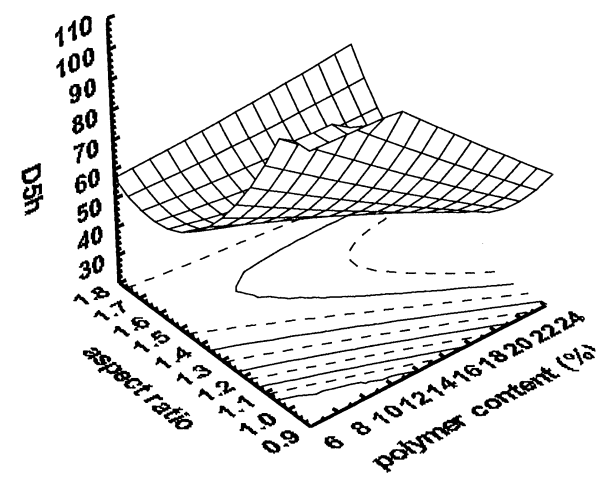

b

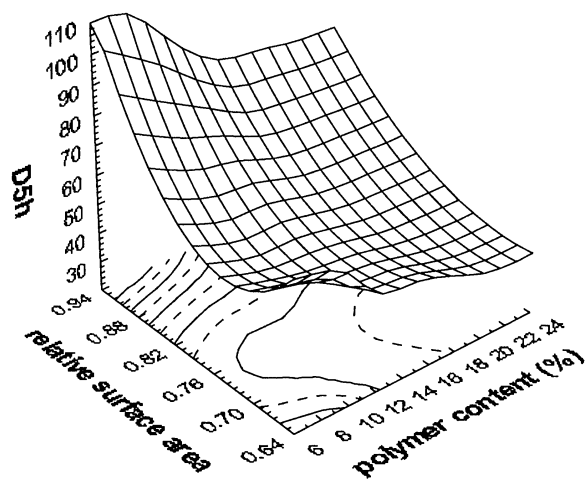

FIG. 3. Response surface and contour plot of (a) $D_{5 h}$ vs. polymer content vs. aspect ratio; (b) $D_{5 h}$ vs. polymer content vs. relative surface area for samples prepared with Carbopol 971P.

a

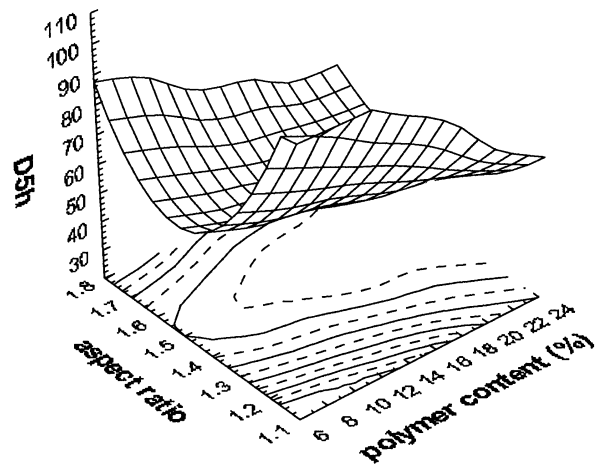

b

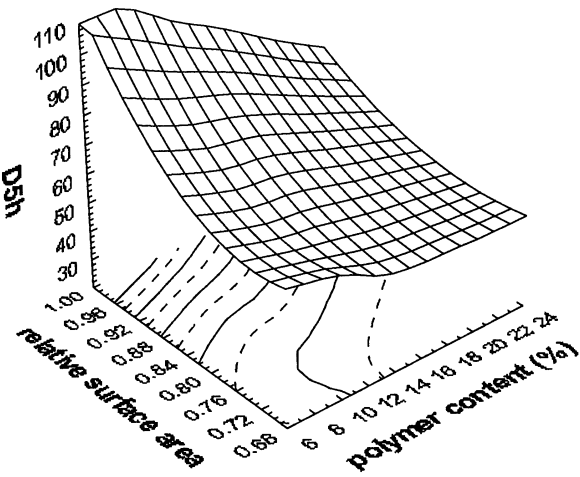

FIG. 4. Response surface and contour plot of (a) $D_{5 h}$ vs. polymer content vs. aspect ratio; (b) $D_{5 h}$ vs. polymer content vs. relative surface area for samples prepared with Carbopol $71 \mathrm{G}$.
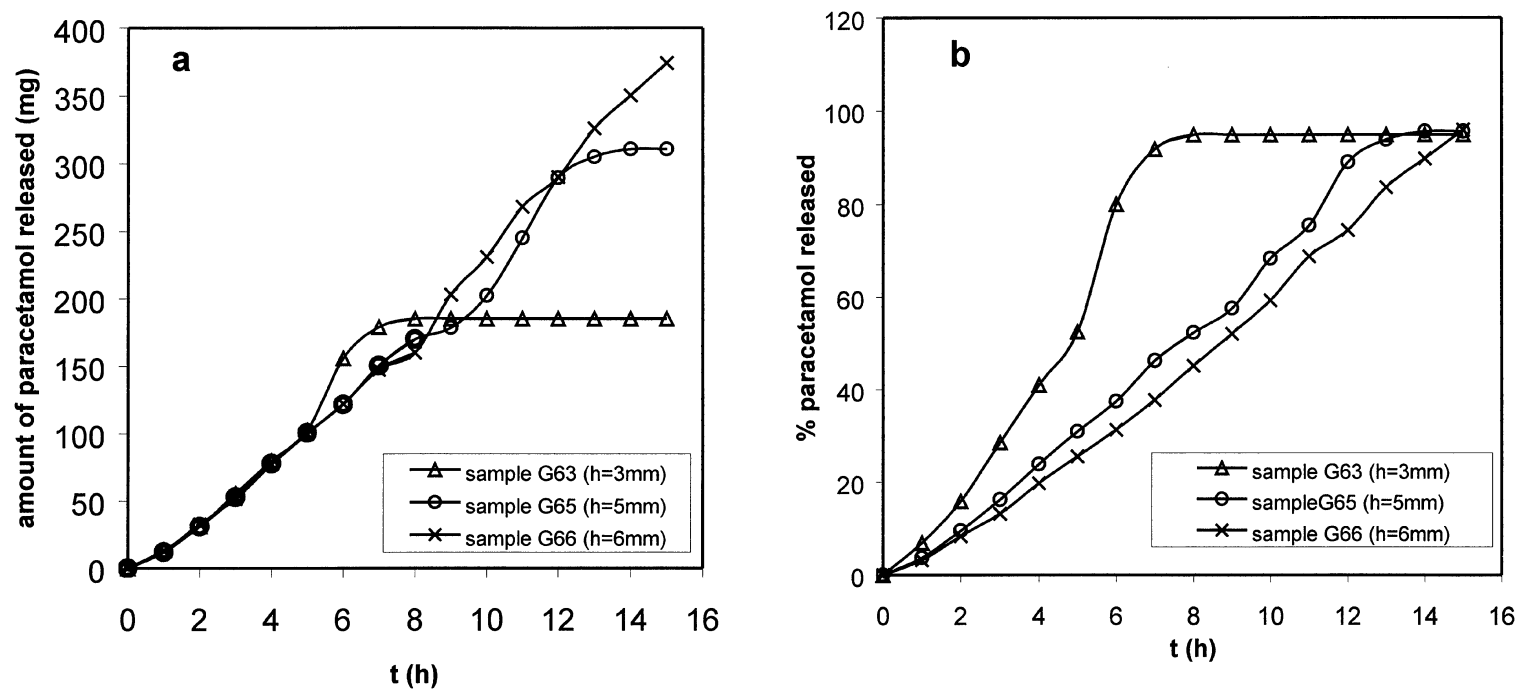

FIG. 5. Effect of the initial tablet height on the (a) resulting absolute amounts of drug released; (b) resulting relative amounts (\%) of drug released from Carbopol $71 \mathrm{G}$ matrix tablets of various height. $\triangle: 3 \mathrm{~mm}, 0: 5 \mathrm{~mm} ; \times: 6 \mathrm{~mm}$. 
TABLE 5

Predicted and observed values of $D_{5 h}$ for samples with different tablet heights

\begin{tabular}{|c|c|c|c|c|c|}
\hline \multirow[b]{2}{*}{ Sample } & \multicolumn{4}{|c|}{ Predicted $\mathrm{D}_{5 \mathrm{~h}}$ values $(\% \mathrm{PE})$} & \multirow{2}{*}{$\begin{array}{c}\text { Experimentally } \\
\text { observed } \mathrm{D}_{5 \mathrm{~h}} \text { values }\end{array}$} \\
\hline & Based on $\mathrm{d}$ & Based on $\mathrm{r} / \mathrm{h}$ & Based on $\mathrm{P}$ & Based on $\mathrm{P} / \mathrm{V}$ & \\
\hline G6 & $22.76(18.42)$ & $22.82(18.21)$ & $16.25(41.76)$ & $25.56(8.39)$ & 27.90 \\
\hline G63 & $22.76(56.70)$ & $97.16(84.86)$ & $24.45(53.48)$ & $60.39(14.90)$ & 52.56 \\
\hline G65 & $22.76(53.62)$ & $52.88(70.80)$ & $14.36(53.62)$ & $27.10(12.47)$ & 30.96 \\
\hline G66 & $22.76(52.93)$ & $125.31(389.88)$ & $12.04(52.93)$ & $28.31(10.67)$ & 25.58 \\
\hline G8 & $34.82(8.91)$ & $35.20(10.10)$ & $21.73(32.03)$ & $35.38(10.67)$ & 31.97 \\
\hline
\end{tabular}

where $\mathrm{Y}_{\mathrm{obs}}$ is the experimentaly observed value of the response variable and $Y_{\text {pred }}$ is the value predicted according to the proposed mathematical model. The internal predictability was estimated from the results used to develop the mathematical model (samples G6 and G8). The external predictability was anticipated using data from the additional samples of tablets with various tablet height (G63, G65, G66). The predicted values of percent of drug released after $5 \mathrm{hr}$ of investigation calculated according to the proposed mathematical models and percent prediction error calculated for each example, as well as the observed values, are presented in Table 5. Good agreement between theory and experiment was observed in the mathematical model based on relative surface area as the parametar describing tablet dimensions, quantified by a percent prediction error ranging from 8.39 to $14.90 \%$

\section{CONCLUSION}

Our results indicate that it may be possible to control the rate of drug release by modifying the proportion of carbomer in tablets and tablet dimensions. The kinetics of drug release depended on the type of polymer, as well as on the polymer content and tablet geometry. Although chemically identical, Carbopol 971P and Carbopol 71G exhibit different drug release patterns due to the technological modifications involved in the manufacture of Carbopol 71G. The present results suggest that the release kinetics are mostly regulated by a swelling-controlled diffusion process, because most of the drug is released while a large portion of the polymer matrix remains intact.

Both the content of polymer and tablet geometry and dimensions should be evaluated to optimize the formulation with the targeted drug release pattern. Although the tablet diameter seems to be the prevalent tablet size parameter determining the drug release rate, we found that relative surface area is a more relevant term for comparison of tablets of varying height, which also is expected to be useful for comparison of tablets of different shape. The practical benefit of these simulations is to optimize the geometry and dimensions of a controlled release device and reduce the number of experiments involved in the development of new controlled release dosage forms.

\section{REFERENCES}

Amidon, L. G., and Lobenberg, R. 2000. Modern bioavailability, bioequivalence and biopharmaceutics classification system. New scientific approaches to international regulatory standards. Eur. J. Pharm. Biopharm. 50:3-12.

Box, G. E. P., Hunter, W. G., and Hunter, J. S. 1978. Statistic for Experimenters. An Introduction to Design, Data Analysis and Model Building, New York: John Wiley \& Sons.

Channer, K. S., and Virjee, J. P. 1986. The effect of size and shape of tablets on their esophageal transit. J. Clin. Pharmacol. 26:141-146.

Fereira, D. C., Prista, L. V., Morgado, R. M., and Lobo, J. M. S. 1995. Sustainedrelease tablet containing oxazepam: Study and design. Drug Dev. Ind. Pharm. 21:591-604.

Huang, L. L., and Schwartz, B. J. 1995. Studies on drug release from a carbomer tablet matrix. Drug Dev. Ind. Pharm. 21:1487-1501.

Karasulu, H. Y., Ertan, G., and Kose, T. 2000. Modeling of theophylline release from different geometrical erodible tablets. Eur.J.Pharm. Biopharm. 49:177182.

Khan, G. M., and Zhu, J. B. 1999. Studies on drug release kinetics from ibuprofen-carbomer hydrophilic matrix tablets: Influence of co-excipients on release rate of the drug. J. Control. Rel. 57:197-203.

Kim, C. J. 1995. Compressed donut-shaped tablets with zero-order release kinetics. Pharm. Res. 12:1045-1048.

Montgomery, D. 1997. Design and Analysis of Experiments, 4th Ed. New York: John Wiley \& Sons.

Perez-Marcos, B., del Cano, O., Gomez-Amoza, J. L., Martinez-Pacheco, R., Souto, C., and Concheiro, A. 1995. Design and biopharmaceutical evaluation of atenolol matrix tablets prepared with carbomer 934. STP Pharma 5:105109.

Ritger, P., and Peppas, N. 1987. A simple equation for description of solute release II: Fickian and anomalous release from swellable devices. J. Control. Rel. 5:37-42.

Siepmann, J., Ainaoui, A., Vergnaud, J. M., and Bodmeier, R. 1998. Calculation of the dimensions of drug-polymer devices based on diffusion parameters. J. Pharm. Sci. 87:827-832.

Siepmann, J., Kranz, H., Peppas, N. A., and Bodmeier, R. 2000. Calculation of the required size and shape of hydroxypropylmethylcellulose matrices to achieve desired drug release profiles. Int. J. Pharm. 201:151-164.

Siepmann, J., Streubel, A., and Peppas, N. A. 2002. Understanding and predicting drug delivery from hydrophilic matrix tablets using the "sequential layer" model. Pharm. Res. 19:306-314.

Reynolds, T. D., Mitchell, S. A., and Balwinski, K. M. 2002. Investigation of the effect of tablet surface area/volume on drug release from hydroxypropylmethylcellulose controlled-release matrix tablets. Drug Dev. Ind. Pharm. 28:457-466.

Wilson, G. C. 1998. Gastrointestinal transit and drug absorption. In Biopharmaceutics Classification System and In Vitro-In Vivo Correlations, APV Kurs. Frankfurt, Germany, 23-25 March 1998. 